\title{
Inhibition of tubulointerstitial fibrosis by pentoxifylline is associated with improvement of vascular endothelial growth factor expression
}

\author{
Qiu-gen $\mathrm{ZHOU}^{1}$, Fa-lei ZHENG ${ }^{2, *}$, Fan-fan $\mathrm{HOU}^{1, *}$ \\ ${ }^{1}$ Division of Nephrology, Southern Medical University Nanfang Hospital, Guangzhou 510515, China; ${ }^{2}$ Division of Nephrology, Peking Union Medical Col- \\ lege Hospital, Peking Union Medical College and Chinese Academy of Medical Sciences, Beijing 100730, China
}

\begin{abstract}
Aim: Recent information indicates that pentoxifylline (PTX) has the ability to suppress inflammation and profibrotic cell proliferation. In this study, we investigated the effect of PTX on tubulointerstitial fibrosis and the expression of vascular endothelial growth factor (VEGF) in a rat model of obstructive nephropathy.

Methods: Wistar rats with left ureteral ligation were divided into control and PTX-treated groups. The histopathologic degree of tubulointerstitial fibrosis was scored with PAS and Masson-stained sections. The protein and mRNA for vascular endothelial growth factor (VEGF) were semiquantitatively measured with immunohistochemistry and RT-PCR. The protein for transforming growth factor $\beta 1$ (TGF $\beta 1$ ) and hypoxia-induced factor 1 alpha (HIF-1a) was determined by Western blot.

Results: Compared with the control group, PTX treatment reduced fibrosis scores at $\mathrm{d} 7$ and $\mathrm{d} 14(P<0.05)$. The reduction was accompanied by inhibited expression of transforming growth factor-beta 1 (TGF $\beta 1$ ), a key cytokine in tubulointerstitial fibrogenesis $(P<0.01)$. Meanwhile, VEGF protein and mRNA in the kidney were increased in the PTX-treated group compared with the control group $(P<0.01)$. PTX up-regulated expression of VEGF mRNA in a dose- and time-dependent manner in cultured HK-2 cells $(P<0.01)$. However, expression of HIF-1a (a key transcription factor for VEGF gene expression) was unchanged by PTX treatment. PTX prolonged the half-life of VEGF mRNA by a 1.07-fold increase.

Conclusions: PTX inhibited tubulointerstitial fibrosis in a rat model of obstructive nephropathy while preventing loss of VEGF. PTX up-regulated expression of VEGF mRNA through stabilization of its mRNA in cultured renal tubular epithelial cells.
\end{abstract}

Keywords: tubulointerstitial fibrosis; vascular endothelial growth factor; pentoxifylline

Acta Pharmacologica Sinica (2009) 30: 98-106; doi: 10.1038/aps.2008.11; published online 15th December 2008

\section{Introduction}

Tubulointerstitial fibrosis is closely correlated with the progression of chronic renal disease (CKD) and is a common final pathway leading to end-stage renal disease ${ }^{[1]}$. Recently, the role of peritubular capillary loss in the development of tubulointerstitial fibrosis has been increasingly recognized $^{[2,3]}$. A close relationship between progressive tubulointerstitial fibrosis and the loss of peritubular capillary has been demonstrated in patients with $\mathrm{CKD}^{[4]}$ and in animal models of $\mathrm{CKD}^{[s-9]}$. Furthermore, loss of peritubular

* Correspondence to Prof Fa-lei ZHENG or Prof Fan-fan HOU.

E-mail pumckidney@hotmail.com; ffhou@fimmu.edu.cn

Received 2008-07-29 Accepted 2008-10-20 capillary has been associated with local vascular endothelial growth factor (VEGF) depletion and possibly the downregulation of VEGF in renal tubular epithelial cells ${ }^{[6,7]}$. Treatment with VEGF in a rat model of remnant kidneys inhibited loss of peritubular capillary while improving renal function and peritubular capillary endothelial cell proliferation ${ }^{[9]}$. This information indicates that preventing the loss of peritubular capillary may help to slow the progression of tubulointerstitial fibrosis and CKD.

VEGF was initially identified as a potent endothelial cell mitogen. It acts via specific receptors, such as VEGF receptor-1 (Flt-1) and VEGF receptor-2 (Flk-1), to promote angiogenesis and increase vascular permeability ${ }^{[10]}$. Hypoxiainduced factor-1 (HIF-1) is the most important transcrip- 
tion factor driving VEGF mRNA expression. HIF-1 $a$ stabilization and accumulation is essential for HIF-1 to exert its function $^{[11]}$. VEGF is notable for its broad involvement in cellular function. It has been associated with tumor growth and wound healing, among other processes. In the kidney, VEGF is considered to be critical for endothelial cell repair during the course of renal disease ${ }^{[12]}$. Furthermore, VEGF has been shown to induce nephrogenesis and vasculogenesis in all renal tubular epithelial cells ${ }^{[13,14]}$. VEGF induced a proliferative response while preventing hydrogen peroxideinduced apoptosis and necrosis in renal tubular epithelial cells $^{[15]}$. This suggests that VEGF may act as a survival factor for the tubular epithelium. Substantial evidence shows that VEGF is able to support the growth of renal epithelia and mediate kidney vascularization through an autocrine and paracrine pathway $^{[16]}$.

Pentoxifylline (PTX) is a methylxanthine derivative and a nonspecific type 4 phosphodiesterase inhibitor. It is used clinically to treat patients with peripheral vascular diseases ${ }^{[17]}$. Its pharmacological mechanisms are not completely understood. PTX has been shown to reduce production of collagen, expression of interleukin-6 and expression of transforming growth factor-beta 1 (TGF $\beta 1)$ in rat hepatic stellate ${ }^{[18]}$. PTX also inhibits the proliferation of cultured lymphocytes, fibroblasts, and mesangial cells while reducing the production of extracellular matrix proteins ${ }^{[19]}$. In an in vivo study, PTX attenuated experimental cyclosporine nephropathy and mesangial proliferative glomerulonephritis ${ }^{[20]}$. PTX slowed chronic renal disease progression in rat model of a remnant kidney ${ }^{[21]}$ and attenuated tumor necrosis factor- $\alpha$ (TNF- $\alpha$ )mediated renal injury ${ }^{[22]}$. These studies suggest that PTX has the ability to suppress inflammation, profibrotic cell proliferation and fibrosis. However, whether PTX has an inhibitory effect on tubulointerstitial fibrosis and the expression of VEGF remains unclear.

In the present study, we used a rat model of obstructive nephropathy to test whether PTX could ameliorate tubulointerstitial fibrosis and up-regulate the expression of VEGF in tubulointerstitium. Our data showed that PTX inhibited tubulointerstitial fibrosis while preventing loss of VEGF. In a cultured HK-2 tubular epithelial cell line, PTX directly upregulated the expression of VEGF mRNA by stabilization of its mRNA. Our findings may have implication for clinical use of PTX in patients with chronic tubulointerstitial injury.

\section{Materials and methods}

Reagents PTX, actinomycin D, and an assay kit to detect blood urea nitrogen and serum creatinine were purchased from Sigma (St Louis, MO, USA). Rabbit anti-VEGF, antiTGF $\beta 1$ and anti-HIF- $1 \alpha$ as well as goat anti-GAPDH polyclonal antibody and ECL were obtained from Santa Cruz Biotechnology (Santa Cruz, CA, USA). The Histostain ${ }^{\mathrm{TM}}$ plus kit was from Zymed Laboratories (South San Francisco, CA, USA). The TRlzol reagent was from Life Technologies/ BRL (Rockville, MD, USA). M-MLV reverse transcriptase and Taq polymerase were purchased from Promega (Madison, WN, USA). BCA protein assay kits were purchased from Biyuantian (Shanghai, China).

Experimental design Female Wistar rats (190 to 210 g, Grade III, Peking Union Medical College Hospital, certificate SYXK 11-00-0041) were divided into two 1:2 matched groups: a sham operation group and a unilateral ureteral obstruction (UUO) group. The animals were housed in individual cages under constant temperature and humidity on a 12-h light/dark cycle. Food and water were provided ad libitum. Protocols for animal experimentation and maintenance were approved by the Animal Ethics Committee at our institute and carried out in accordance with institutional guidelines. With anesthesia with sodium pentobarbital (40 $\mathrm{mg} / \mathrm{kg}$, intraperitoneally), the left ureter was ligated with silk sutures at two points and was cut between the ligatures. The UUO rats were randomly divided into two groups: one group receiving PTX (50 mg/kg body weight per day by oral gavages, $n=9$ ) from d 1 to d 14 after ligation, the other group receiving vehicle (drinking water only, $n=9$ ) as control. Three rats from each group were sacrificed on $\mathrm{d} 3,7$, and 14 after UUO. Three sham-operated rats were also sacrificed at each time point to serve as negative controls. Kidneys were decapsulated and divided into several parts. One part was fixed in $10 \%$ neutral buffered formalin and processed for histological analysis. Others were dissected to isolate the cortex, which was quickly frozen in liquid nitrogen and stored at $-70{ }^{\circ} \mathrm{C}$ for protein and total RNA extraction. Blood was collected for serum creatinine and blood urea nitrogen measurements according to the protocol of the commercial kit.

Histologic examinations Kidneys were removed, fixed in $10 \%$ neutral formalin, embedded in paraffin, sectioned (2.0-3.0 $\mu \mathrm{m}$ thickness), stained with periodic acid-Schiff (PAS) and Masson trichrome using routine procedures. Evaluation of tubulointerstitial fibrosis was performed. In each section, 40 randomly selected fields were examined under $\times 400$ magnification for assessment of the degree of tubulointerstitial fibrosis ${ }^{[21]}$ (the semiquantitative score for tubulointerstitial fibrosis). The degree of tubulointerstitial fibrosis was scored in PAS- and Masson-stained sections as follows: 0 , normal tubules and no fibrosis; 1 , mild interstitial 
fibrosis, with almost normal tubules; 2 , more severe interstitial fibrosis, with some atrophic tubules and thickness of the tubular basement; 3, marked interstitial fibrosis, with diffuse atrophic tubules and thickness of the tubular basement. The semiquantitative scores for the tubulointerstitial fibrosis of the 40 randomly selected fields were added up. Tubulointerstitial fibrosis was examined in each rat and averaged for the three rats from each group.

Immunohistochemical examinations Indirect immunoperoxidase staining with a Histostain ${ }^{\mathrm{TM}}$-plus kit was performed to assess the expression of VEGF. Sections 2.0-3.0$\mu \mathrm{m}$ thick were mounted on a poly- $L$-lysine coated slide, deparaffinized with xylene and rehydrated. The sections were microwaved in $10 \mathrm{mmol} / \mathrm{L}$ citrate buffer $(\mathrm{pH} \mathrm{6.0)}$ ) at $100{ }^{\circ} \mathrm{C}$ for $5 \mathrm{~min}$ to unmask the antigen. After blocking the endogenous peroxidase, they were subsequently incubated with primary antibodies (1:50 diluted with $1.5 \%$ bovine serum albumin for VEGF) at $4{ }^{\circ} \mathrm{C}$ overnight. The reactions on the sections were detected with peroxidase substrate containing AEC. The specific antibodies were omitted in sections to serve as negative controls. All sections were counterstained with hematoxylin. The degree of VEGF expression (the staining area) was evaluated with image analysis software. The result was expressed as the percentage of the staining area in the selected field under $\times 400$ magnification. To exclude the effects of glomeruli on measured values, areas containing glomeruli in selected fields were avoided.

Cell culture Human proximal tubular epithelial cells HK-2 (from Dr Xiong-zhong RUAN, Royal Free and University College Medical School, UK) were cultured and maintained in Dulbecco's modified Eagle's medium/ Ham's F-12 containing 10\% fetal bovine serum as previously described ${ }^{[23]}$. All studies were carried out in overnight serum-deprived cells at $90 \%-100 \%$ confluency.

Reverse transcription-PCR Total RNA was isolated from the cortex of the ligated kidneys or cultured cells using the TRIzol reagent according to the manufacturer's instructions. First-strand cDNA was made from total RNA using the M-MLV transcriptase. PCR was performed in a DNA Thermal Cycler with the primers mentioned below. Oligonucleotide primers were designed to amplify VEGF mRNA and identify unique VEGF isoforms ${ }^{[24]}$. The sequence of the sense primer was 5'-GGACATCTTCCAGGAGTACC-3', and the sequence of the antisense primer was 5'-GTTCCCGAAACCCTGAGG-3'. The sizes expected for $\mathrm{VEGF}_{120}$, $\mathrm{VEGF}_{164}$ and $\mathrm{VEGF}_{188}$ are $438 \mathrm{bp}, 306 \mathrm{bp}$, and $504 \mathrm{bp}$, respectively. The GAPDH mRNA was chosen as an internal control. The sequence of rat GAPDH sense primer was 5'CATGACCACAGTCCATGCCATC-3', and the antisense primer was 5'-CACCCTGTTGCTGTAGCCATATTC-3'. The expected size for GAPDH is $451 \mathrm{bp}$. The sequence of the human GAPDH sense primer was 5'-CCACCCATGGCAAATTCCATGGCA-3'. The sequence of the human GAPDH antisense primer was 5'TGCTAAGCAGTTGTTGGTGCAGGA-3', with an expected size of $323 \mathrm{bp}$. Reactions were incubated at $95^{\circ} \mathrm{C}$ for $5 \mathrm{~min}$, followed by the indicated cycles of $60 \mathrm{~s}$ at $94^{\circ} \mathrm{C}, 60 \mathrm{~s}$ at $55.5^{\circ} \mathrm{C}$ for $\operatorname{VEGF}\left(58.5^{\circ} \mathrm{C}\right.$ for GAPDH), $60 \mathrm{~s}$ at $72^{\circ} \mathrm{C}$, and a final extension at $72^{\circ} \mathrm{C}$ for 10 min. There were 30 cycles of PCR for VEGF and 28 cycles for GAPDH. The PCR products were electrophoresed in $2 \%$ agarose and determined by the signal intensity (UVI soft UVIband windows application V97.04). The results were normalized against the signal intensity of GAPDH.

Western blot analysis Kidney tissues or cultured cells were homogenized in RIPA lysis buffer (1\% NP40, $0.1 \%$ SDS, $100 \mu \mathrm{g} / \mathrm{mL}$ phenylmethylsulfonyl fluoride, $0.5 \%$ sodium deoxycholate, in PBS) on ice. The supernatants were collected after centrifugation at $12000 \times g$ at $4{ }^{\circ} \mathrm{C}$ for $20 \mathrm{~min}$. Protein concentration was determined using a BCA protein assay kit, and whole lysates were mixed with an equal amount of $2 \times \mathrm{SDS}$ loading buffer ( $125 \mathrm{mmol} / \mathrm{L}$ Tris- $\mathrm{HCl}, 4 \% \mathrm{SDS}$, $20 \%$ glycerol, $100 \mathrm{mmol} / \mathrm{L} \mathrm{DTT}$, and $0.2 \%$ bromophenol blue). Samples were heated at $100{ }^{\circ} \mathrm{C}$ for $5 \mathrm{~min}$ and were separated on SDS-polyacrylamide gels. The separated proteins were then transferred to a PVDF membrane. The membrane blots were first probed with a primary antibody. After incubation with horseradish peroxidase-conjugated second antibody, autoradiograms were prepared using the enhanced chemiluminescent system to visualize the protein antigen. The signals were recorded by X-ray film and determined by the signal intensity (UVI soft UVI band Windows application V97.04). The primary antibodies that we used included anti-TGF $\beta 1$ (1:400), anti-GAPDH (1:500) and anti-HIF$1 \alpha(1: 400)$. GAPDH was chosen as the internal control. The results were normalized against the signal intensity of GAPDH.

mRNA stability assay Overnight serum-deprived HK2 cells were treated with PTX of $0.5 \mathrm{~g} / \mathrm{L}$ for $24 \mathrm{~h}$ before treatment with $5 \mu \mathrm{mol} / \mathrm{L}$ actinomycin D. VEGF mRNA levels at $0,30,60,90,120$, and $240 \mathrm{~min}$ after addition of actinomycin D were assessed by RT-PCR as mentioned above. The half-life $\left(t_{1 / 2}\right)$ of the VEGF mRNA was calculated from the slope of the logarithmic plot of relative mRNA levels versus time of decay, using a linear regression analysis ${ }^{[25]}$.

Statistical analyses All data were expressed as means \pm SD. Statistical analyses were carried out using the software SSPS 11.5 for Windows. The statistical significance was evaluated by one-way ANOVA. The statistical significance 
between two groups was evaluated by $t$-test. Values of $P<0.05$ were considered to be statistically significant.

\section{Results}

PTX improved histopathologic changes in UUO rats After ureteral ligation, rats developed hydronephrosis with progressive dilation of the renal pelvis and thinning of the renal cortex. Serum creatinine and blood urea nitrogen levels remained unchanged at all time points (data not shown). Renal tubular damage after UUO increased progressively from $\mathrm{d} 3$ to 14, which was characterized by dilation with flattened epithelium at $\mathrm{d} 3$, atrophy and thickness of tubular basement membrane at $\mathrm{d} 7$ and destruction of tubules at d 14. In parallel, a progressive expansion of the interstitial space and fibrosis gradually developed, as revealed by PAS and Masson trichrome staining (Figure 1). There was no difference in tubulointerstitial fibrosis between the UUO group and the PTX-treated group at d $3(P=0.67)$. Meanwhile, the tubulointerstitial fibrosis score was reduced by $36.6 \% \pm 3.7 \%$ at $\mathrm{d} 7$ and $28.3 \% \pm 2.7 \%$ at $\mathrm{d} 14$ in PTX-treated group compared with the score of $65.6 \pm 4.5$ at $\mathrm{d} 7$ and $96.7 \pm 7.0$ at $\mathrm{d} 14$ in the UUO group $(P<0.05)$ (Figure 2).

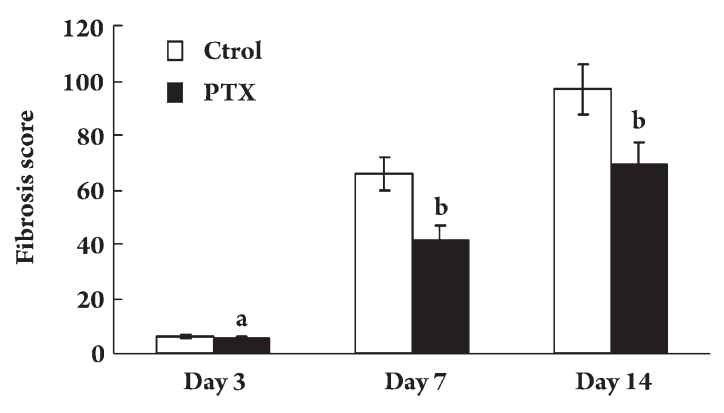

Figure 2. Semiquantitative tubulointerstitial fibrosis score in control and PTX-treated group. Compared with control group, the fibrosis score was reduced in PTX treated group at day 7 and day 14. The values were expressed as mean $\pm S D$ of 3 independent experiments. ${ }^{\mathrm{a}} \mathrm{P}>0.05,{ }^{\mathrm{b}} \mathrm{P}<0.05$ vs control group.

\section{PTX inhibited expression of TGF $\beta 1$ in UUO rats} There is substantial evidence supporting the central role of TGF $\beta 1$ in the development of tubulointerstitial fibrogenesis ${ }^{[26]}$. To investigate the effect of PTX on expression of TGF $\beta 1$ in the kidney, TGF $\beta 1$ protein was determined with Western blot in UUO rats treated with or without PTX. As shown in Figure 3, the expression of TGF $\beta 1$ protein was significantly upregulated in the UUO group. Compared

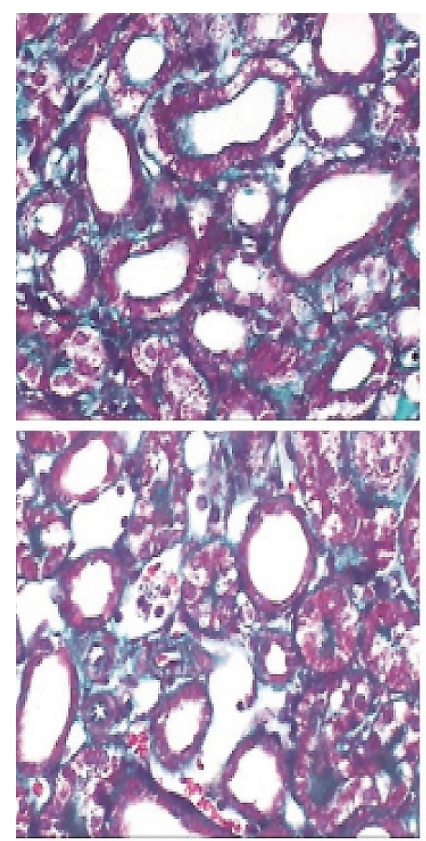

Day 3

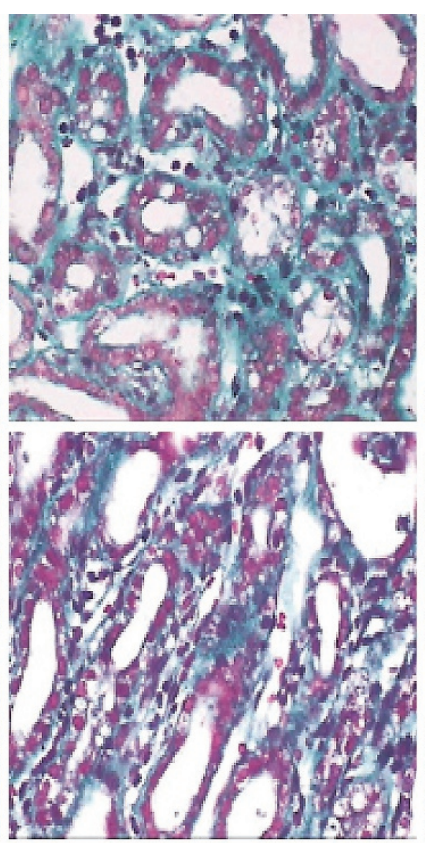

Day 7

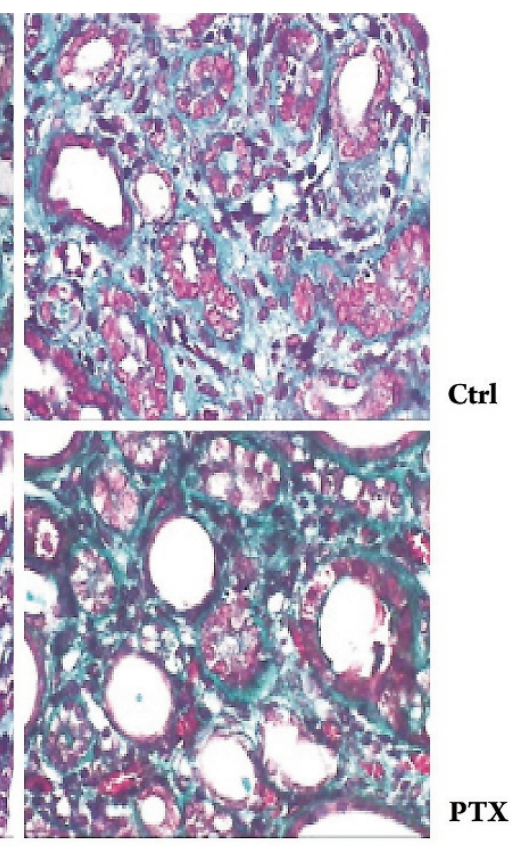

Day 14

Figure 1. Representative histopathology in UUO rats treated with or without PTX ( Massone stain, $\times 400$ ). Day 3 after ligation, renal tubular dilation with flattened epithelium and without atrophy or thickness of tubular basement membrane accompanied with few fibrosis in interstitial space. Day 7 after ligation, some tubular atrophy and thickness of tubular basement membrane accompanied with moderate fibrosis in interstitial space. Day 14 after ligation, diffused tubular atrophy and thickness of tubular basement membrane accompanied with severe fibrosis in interstitial space. 
A

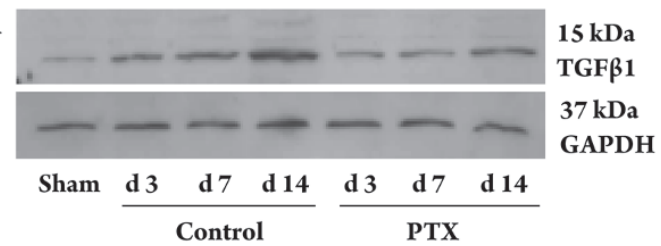

B

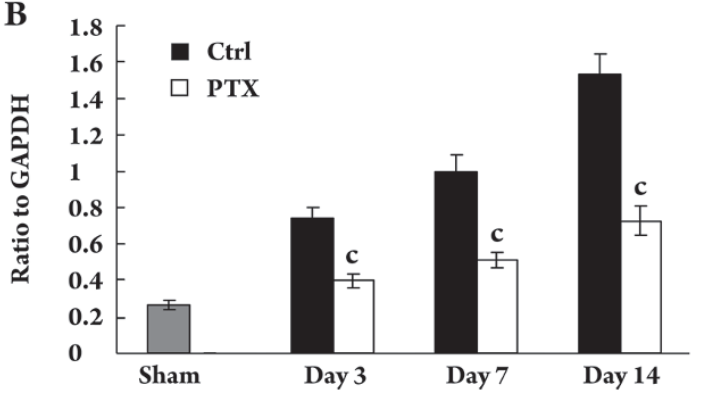

Figure 3. Expression of TGF $\beta 1$ protein in kidney tissue. (A) Representative western blot of TGF $\beta 1$ in sham and UUO rat treated with or without PTX. (B) Compared with control group, PTX treatment inhibited the increased expression of TGF $\beta 1$. The data was shown as ratio of TGF $\beta 1$ density to GAPDH density. The values were expressed as mean \pm SD of 3 independent experiments. ${ }^{c} P<0.01 \mathrm{vs}$ control.

with the sham group, the UUO group was $74.1 \% \pm 5.92 \%$ at $\mathrm{d} 3,99.9 \% \pm 8.97 \%$ at $\mathrm{d} 7$ and $153.7 \% \pm 7.51 \%$ at $\mathrm{d} 14$. PTX treatment reduced TGF $\beta 1$ expression by $45.8 \% \pm 4.4 \%$ at $\mathrm{d} 3$, $48.8 \% \pm 4.9 \%$ at $\mathrm{d} 7$ and $52.6 \% \pm 5.4 \%$ at $\mathrm{d} 14$ compared with the UUO group at the same time points $(P<0.01)$.

PTX improved reduced-expression of VEGF in UUO rats To examine the effect of PTX on VEGF expression in kidney, its protein and mRNA levels were measured in UUO rats treated with or without PTX. A semi-quantitative study with immunohistochemistry revealed a VEGF protein of $78.5 \% \pm 2.9 \%$ at $\mathrm{d} 3,46.5 \% \pm 5.9 \%$ at $\mathrm{d} 7$ and $27.4 \% \pm 5.5 \%$ at $\mathrm{d} 14$ in the control group (Figure 4). There were three isoforms of VEGF mRNA in renal tissue (Figure 5), consistent with previous reports ${ }^{[23]}$. VEGF mRNA level was $52.3 \% \pm 5.1 \%$ at $\mathrm{d} 3,18.5 \% \pm 1.9 \%$ at $\mathrm{d} 7$ and $11.8 \% \pm 1.2 \%$ at d 14 in UUO rats. Compared with the control group, PTX treatment increased VEGF protein by $28.8 \% \pm 3.0 \%$ at $\mathrm{d} 7$ and $43.4 \% \pm 4.5 \%$ at $\mathrm{d} 14(P<0.05)$. PTX treatment also increased VEGF mRNA by $114.1 \% \pm 12.1 \%$ at $\mathrm{d} 7$ and $98.3 \% \pm 9.7 \%$ at d $14(P<0.01)$. Therefore, PTX treatment prevented loss of VEGF expression at both the mRNA and protein level at $\mathrm{d} 7$ and $\mathrm{d}$ 14. However, PTX treatment had no effect on VEGF expression at $\mathrm{d} 3$.

PTX up-regulated expression of VEGF mRNA in cultured HK-2 cells Renal tubular epithelial cells have been demonstrated to be an important source of VEGF in the kidney ${ }^{[13,14]}$. To clarify whether PTX directly affects VEGF expression in renal tubular epithelial cells, VEGF mRNA was examined in cultured HK-2 cells treated with or without the indicated concentration of PTX for the indicated time. As shown in Figure 6, HK-2 cells expressed two isoforms of VEGF mRNA. PTX up-regulated expression of VEGF mRNA in a dose- and time-dependent manner $(P<0.01)$.

Effect of PTX on HIF-1a protein level in cultured HK-2 cells HIF-1 is the most important transcription factor driving VEGF mRNA expression. HIF-1 $\alpha$ stabilization and accumulation is essential for HIF-1 to exert its function ${ }^{[11]}$. To examine whether PTX-induced upregulation of VEGF mRNA resulted from activation and transcription of the VEGF gene, and HIF-1 $\alpha$ protein levels were studied in cultured HK-2 cells treated with or without indicated concentration of PTX for $72 \mathrm{~h}$. As shown in Figure 7, PTX treatment had no effect on HIF- $1 \alpha$ protein levels $(P=0.935)$. This result led us to further investigate whether PTX stabilization of VEGF mRNA contributed to increased VEGF levels. After treatment with PTX for $24 \mathrm{~h}$, the cells were treated with actinomycin D for up to $4 \mathrm{~h}$ to prevent further transcription of the VEGF gene. The total RNA was harvested at the indicated time. As shown in Figure 8, the estimated halflife of VEGF mRNA in HK-2 cells was $0.715 \pm 0.094 \mathrm{~h}$. PTX significantly prolonged VEGF mRNA half-life, increasing it by 1.07 fold $(P<0.01)$.

\section{Discussion}

In the present study, the effect of PTX on tubulointerstitial fibrosis and expression of VEGF was tested. Our results showed that PTX inhibited tubulointerstitial fibrosis and prevented loss of VEGF. PTX directly up-regulated expression of VEGF mRNA through prolongation of its half-life in cultured human renal tubular epithelial cells. We provided the evidence that the inhibitory effect of PTX on tubulointerstitial fibrosis was probably associated with improvement of VEGF expression. Consistent with an early report ${ }^{[27]}$, our results provide further evidence to support the protective effect of PTX on kidney disease, while proposing a new mechanism for how PTX exerts its function.

The UUO model is widely used as a model of tubulointerstitial fibrosis. This model is characterized by renal myofibroblast activation, tubular atrophy and interstitial fibrosis with minimal glomerular lesions ${ }^{[28]}$. The obstructed kidneys displayed marked tubulointerstitial injury starting on $\mathrm{d} 3$ after surgery. In agreement with earlier reports ${ }^{[7]}$, inhibition of VEGF expression occurred with development of tubulointerstitial fibrosis. We demonstrated that PTX 


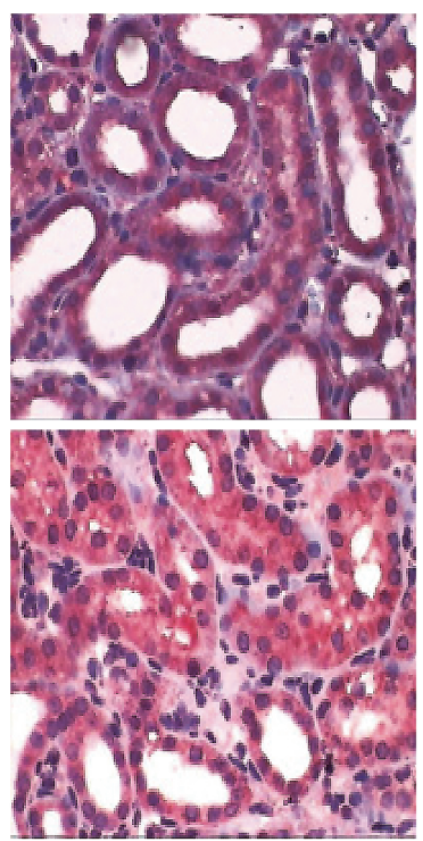

Day 3

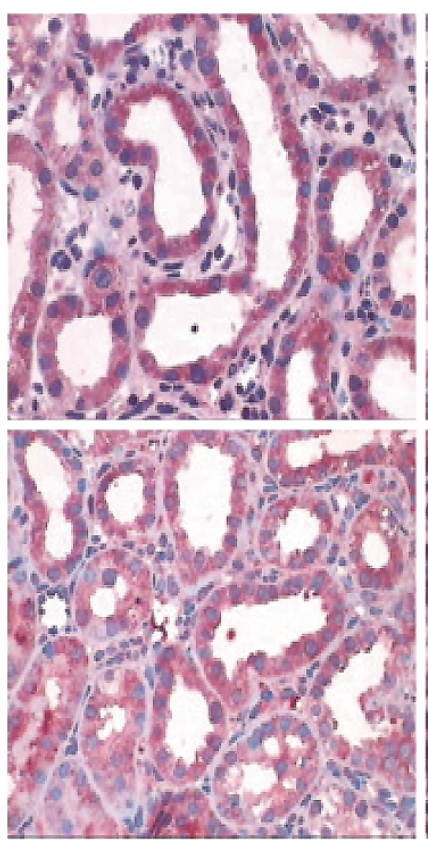

Day 7

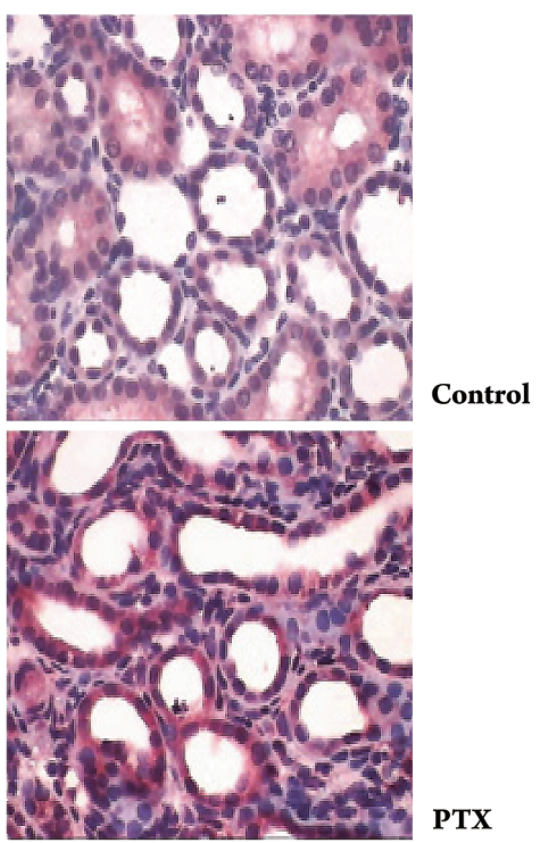

Day 14

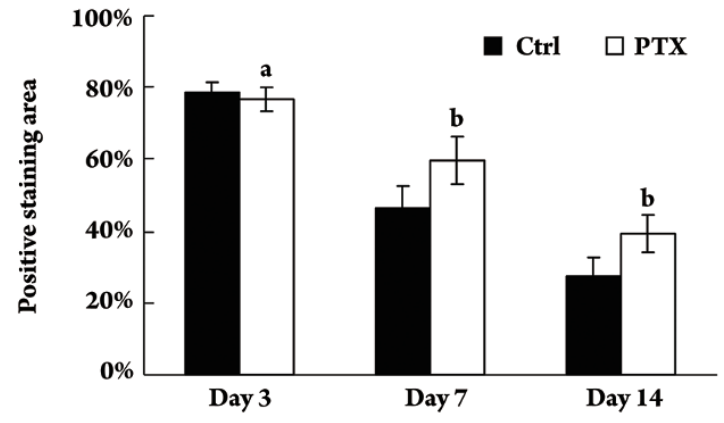

could attenuate histopathologic changes, reduce expression of TGF $\beta 1$ and upregulate expression of VEGF. Inhibition of the expression of TGF- $\beta 1$ by PTX would abolish much of the profibrotic effect of TGF- $\beta 1$. This is due to the central role of TGF $\beta 1$ and its downstream signaling cascades in the activation of the cellular mechanisms that underlie the progression of renal diseases ${ }^{[26]}$. It is likely that inhibition of proliferation of TGF- $\beta 1$-producing cells and inhibition of TGF- $\beta 1$ expression in target cells are mechanisms of PTX. However, this requires further study. VEGF is a survival factor for vascular endothelial cells and plays an important role in the homeostasis of vascular endothelium ${ }^{[2,9]}$. VEGF is also a survival factor for renal tubular epithelial cells and is involved in renal tubule construction ${ }^{[13-15]}$. Supplemention with VEGF improved the interstitial injury mediated by cyclosporine ${ }^{[29]}$. Therefore, modulation of VEGF expression might be a therapeutic approach for prevention of progres- sive renal disease. Renal tubular epithelial cells are an important cellular resource of VEGF in the kidney. Their phenotype usually changes as they transdifferentiate into myofibroblast cells during the late stages of tubulointerstitial injury ${ }^{[28]}$. This change may explain why a PTX-inhibitory effect was not observed until d 7. Prevention of renal tubular epithelial cell function may be one of the targets of PTX. The direct up-regulation of VEGF expression in vitro provides further evidence that PTX may modulate renal tubular epithelial cell function by enhancing VEGF expression to inhibit tubulointerstitial fibrosis.

VEGF expression in human and rat kidneys is confined to podocytes and tubular epithelial cells. There are three isoforms of VEGF in kidney (human $\mathrm{VEGF}_{121}, \mathrm{VEGF}_{165}$, and $\mathrm{VEGF}_{189}$ and rat $\mathrm{VEGF}_{120}, \mathrm{VEGF}_{164}$, and $\left.\mathrm{VEGF}_{188}\right)$. Alternative exon splicing of a single VEGF gene is the basis for this molecular heterogeneity ${ }^{[10]} \cdot \mathrm{VEGF}_{165}$ is a major component, 
A

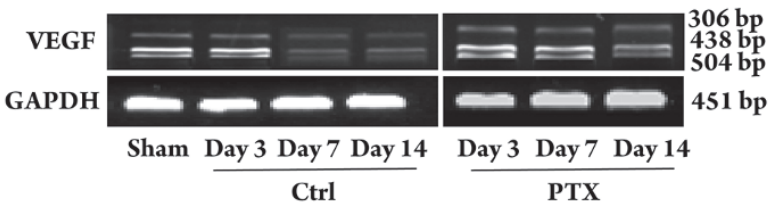

B

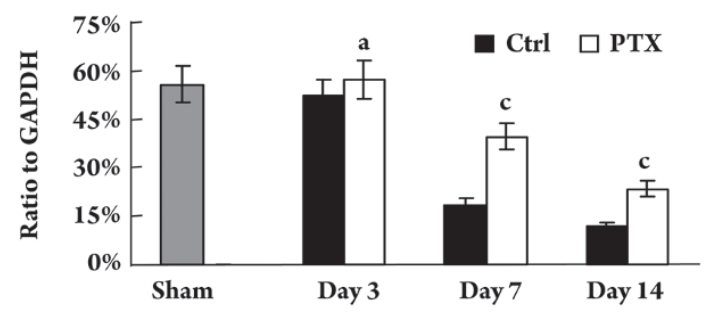

Figure 5. Expression of VEGF mRNA in the kidney determined with RT-PCR in UUO rats treated with or without PTX. (A) Representative electrophoresis of RT-PCR products. $\mathrm{VEGF}_{120}, \mathrm{VEGF}_{164}$, and $\mathrm{VEGF}_{188}$ of 3 VEGF isoforms were expressed in the kidney. (B) Compared with control group, PTX treatment improved the reduced expression of VEGF mRNA at day 7 and day 14 . The band intensities corresponding to the $306 \mathrm{bp}, 438 \mathrm{bp}$, and $504 \mathrm{bp}$ mRNAs were quantified. Data were shown as ratio of VEGF density to that of GAPDH and expressed as mean \pm SD of 3 independent experiments. ${ }^{a} P>0.05,{ }^{c} P<0.01$ vs control group.

and $\mathrm{VEGF}_{121}$ is an intermediate. Both of these are secreted proteins. PTX increased VEGF mRNA levels in cultured renal tubular epithelial cells without affecting HIF-1a, a key transcription factor for the VEGF gene. Our information indicates that PTX may stabilize VEGF mRNA to increase its accumulation. Synthesis of VEGF is regulated at several levels. VEGF is regulated at the level of gene transcription, mRNA stability and mRNA translation ${ }^{[25,30]}$. Post-transcriptional regulation involves $\mathrm{mRNA}$ binding proteins acting on cis elements to alter mRNA stability or the efficiency of translation ${ }^{[30]}$. Considering that VEGF is differentially regulated by hypoxia and TGF $\beta 1$ through different signal pathways $^{[31,32]}$, PTX may induce VEGF gene transcription through HIF-1 $\alpha$ independent pathways including prolonging its mRNA half-life.

In summary, our study shows that PTX inhibits tubulointerstitial fibrosis and improves VEGF expression. Our findings may have implication for clinical use of PTX in patients with chronic tubulointerstitial injury.

\section{Acknowledgement}

Project was supported by National Natural Science Foundation of China (№ 30570854) to Dr Fa-lei ZHENG.

\section{Author contributions}

Fa-lei ZHENG designed research; Qiu-gen ZHOU
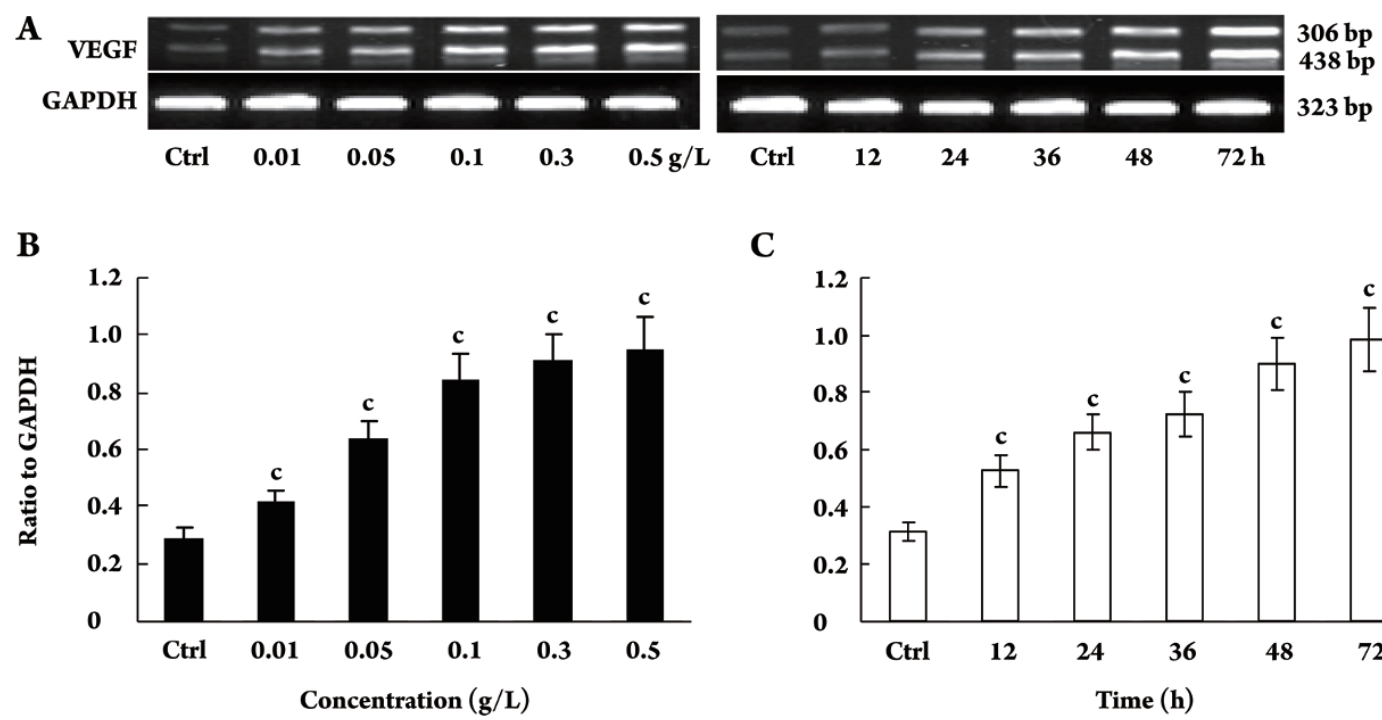

C

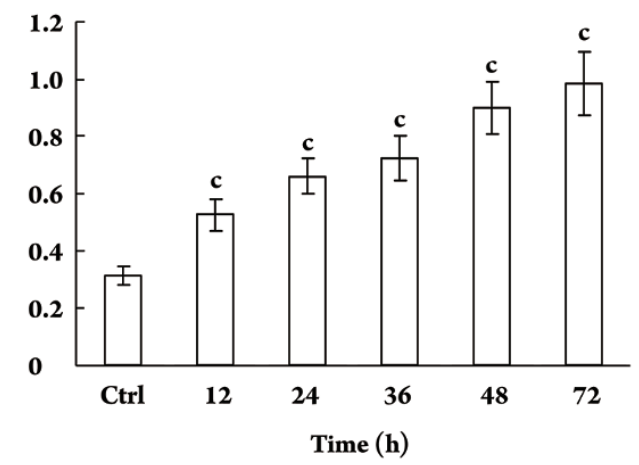

Figure 6. Effect of PTX on expression of VEGF mRNA in cultured HK-2 tubular epithelial cells. (A) Representative electrophoresis of RT-PCR products. VEGF $\mathrm{VE}_{121}$ and VEGF 165 of 2 VEGF isoforms were expressed in cultured HK-2 tubular epithelial cells. (B) Dose-dependent effect of PTX on VEGF mRNA expression. HK-2 cells were treated with indicated concentration of PTX for $72 \mathrm{~h}$. The VEGF mRNA was semiquantatively determined with RT-PCR. The band intensities corresponding to the $306 \mathrm{bp}$ and $438 \mathrm{bp}$ mRNAs were quantified. The data were shown as ratio of VEGF density to that of GAPDH and expressed as mean \pm SD of 3 independent experiments. (C) Time-dependent effect of PTX on VEGF mRNA expression. HK-2 cells were treated with $0.5 \mathrm{~g} / \mathrm{L}$ PTX for the indicated time. ${ }^{c} \mathrm{P}<0.01 v s$ control by ANOVA. 

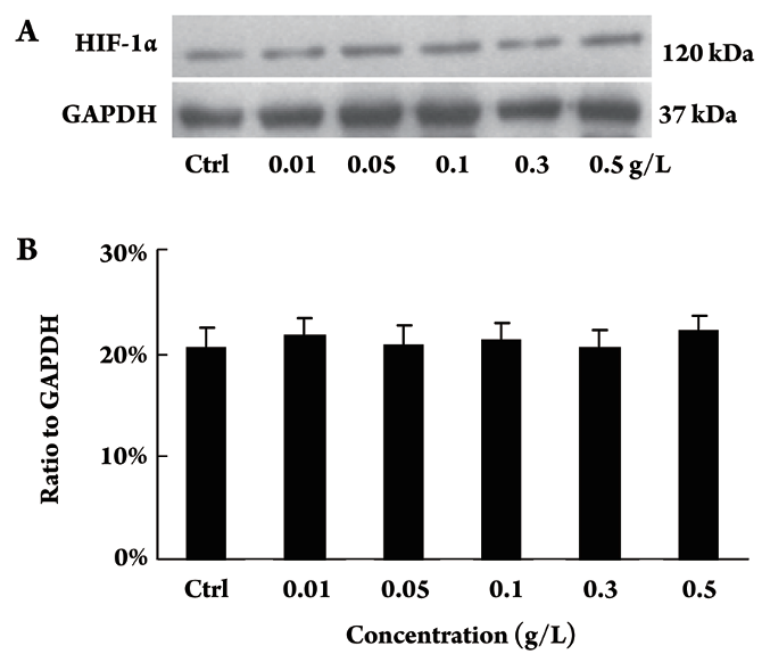

Figure 7. Effect of PTX on expression of HIF-1 $\alpha$ in cultured HK-2 tubular epithelial cells. HK-2 cells were treated with indicated concentration of PTX for $72 \mathrm{~h}$. The expression of HIF-1 1 was determined with Western blot. (A) Representative Western blot of HIF$1 \alpha$ in HK-2 cells. (B) Compared with control, PTX treatment did not change the expression of HIF-1 $\alpha$. The data were shown as ratio of HIF- $1 \alpha$ density to that of GAPDH and expressed as mean \pm SD of 3 independent experiments. $P=0.935 v s$ control by ANOVA.

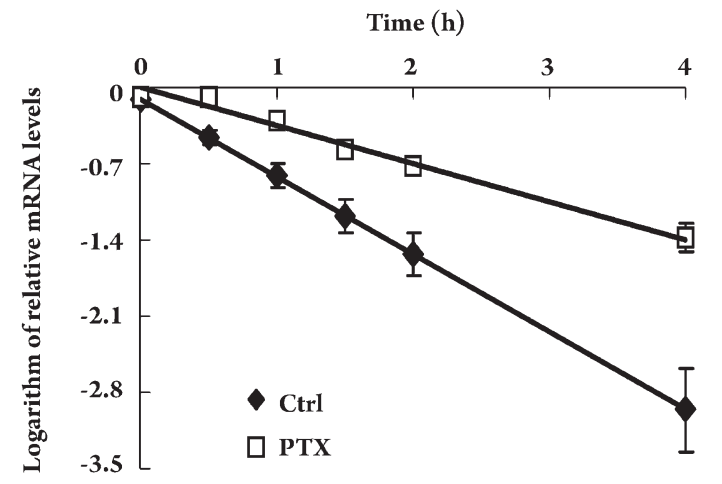

Figure 8. Effect of PTX on VEGF mRNA stability. Overnight serum deprived HK-2 cells were treated with PTX of $0.5 \mathrm{~g} / \mathrm{L}$ for $24 \mathrm{~h}$ before treatment with $5 \mu \mathrm{mol} / \mathrm{L}$ actinomycin D. VEGF mRNA levels at 0 , $0.5,1,1.5,2$, and $4 \mathrm{~h}$ after addition of actinomycin $\mathrm{D}$ were assessed by RT-PCR. The half-life $\left(T_{1 / 2}\right)$ of VEGF mRNA was calculated from the slope of the plot of versus time of decay, using linear regression analysis. The estimated half-life of VEGF mRNA was $0.715 \pm 0.094 \mathrm{~h}$. PTX treatment prolonged the half-life with increase by 1.07 fold. $P<0.01 \mathrm{vs}$ control.

performed research; Fa-lei ZHENG and Qiu-gen ZHOU contributed new analytical tools and reagents; Qiu-gen ZHOU analyzed data; Qiu-gen ZHOU and Fan-fan HOU wrote the paper.

\section{References}

1 Damico G, Ferrario F, Rastaldi MP. Tubulointerstitial damage in glomerular disease: its role in the progression of renal damage. Am J Kidney Dis 1995; 26: 124-32.

2 Kang DH, Kanellis J, Hugo C, Truong L, Anderson S, Kerjaschki D, et al. Role of the microvascular endothelium in progressive renal disease. J Am Soc Nephrol 2002; 13: 806-16.

3 Nangaku M. Chronic hypoxia and tubulointerstitial injury: A final common pathway to end-stage renal failure. J Am Soc Nephrol 2006; 17: $17-25$.

4 Choi YJ, Chakraborty S, Nguyen V, Nguyen C, Kim BK, Shim SI, et al. Peritubular capillary loss is associated with chronic tubulointerstitial injury in human kidney: Altered expression of vascular endothelial growth factor. Hum Pathol 2000; 31: 1491-7.

5 Ohashi R, Kitamura H, Yamanaka N. Peritubular capillary injury during the progression of experimental glomerulonephritis in rats. J Am Soc Nephrol 2000; 11: 47 -56.

6 Yuan HT, Li XZ, Pitera JE, Long DA, Woolf AS. Peritubular capillary loss after mouse acute nephrotoxicity correlates with down-regulation of vascular endothelial growth factor-A and hypoxia-inducible factor-1alpha. Am J Pathol 2003; 163: 2289301.

7 Ohashi R, Shimizu A, Masuda Y, Kitamura H, Ishizaki M, Sugisaki $\mathrm{Y}$, et al. Peritubular capillary regression during the progression of experimental obstructive nephropathy. J Am Soc Nephrol 2002; 13: $1795-805$.

8 Zhang B, Liang X, Shi W, Ye Z, He C, Hu X, Liu S. Role of impaired peritubular capillary and hypoxia in progressive interstitial fibrosis after 5/6 subtotal nephrectomy of rats. Nephrology (Carlton) 2005; 10: 351-7.

9 Kang DH, Hughes J, Mazzali M, Schreiner GF, Johnson RJ. Impaired angiogenesis in the remnant kidney model: II. Vascular endothelial growth factor administration reduces renal fibrosis and stabilizes renal function. J Am Soc Nephrol 2001; 12: 1448-57.

10 Ferrara N. Molecular and biological properties of vascular endothelial growth factor. L Mol Med 1999; 77: 527-43.

11 Zelzer E, Levy Y, Kahana C, Shilo BZ, Rubinstein M, Cohen B. Insulin induces transcription of target genes through the hypoxiainducible factor HIF-1 $\alpha$ /ARNT. EMBO J 1998; 17: 5085-94.

12 Pillebout E, Burtin M, Yuan HT, Briand P, Woolf AS, Friedlander G, et al. Proliferation and remodeling of the peritubular microcirculation after nephron reduction: Association with the progression of renal lesions. Am J Pathol 2001; 159: 547-60.

13 Turo A, Norwood VF, Carey RM, Gomez RA. Vascular endothelial growth factor induces nephrogenesis and vasculogenesis. J Am Soc Nephrol 1999; 10: 2125-34.

14 Villegas G, Lange-Sperandio B, Tufro A. Autocrine and paracrine functions of vascular endothelial growth factor (VEGF) in renal tubular epithelial cells. Kidney Int 2005; 67: 449-57.

15 Kanellis J, Fraser S, Katerelos M, Power DA. Vascular endothelial growth factor is a survival factor for renal tubular epithelial cells. Am J Physiol Renal Physiol 2000; 278: F905-15.

16 Advani A, Kelly DJ, Advani SL, Cox AJ, Thai K, Zhang Y, et al. Role of VEGF in maintaining renal structure and function under normotensive and hypertensive conditions. Proc Natl Acad Sci USA 2007; 104: 14448-53.

17 Magnusson M, Gunnarsson M, Berntorp E, Björkman S, Höglund P. 
Effects of pentoxifylline and its metabolites on platelet aggregation in whole blood from healthy humans. Eur J Pharmacol 2008; 581: 290-5.

18 Hernández E, Correa A, Bucio L, Souza V, Kershenobich D, Gutiérrez-Ruiz MC. Pentoxifylline diminished acetaldehydeinduced collagen production in hepatic stellate cells by decreasing interleukin-6 expression. Pharmacol Res 2002; 46: 435-43.

19 Hewitson TD, Martic M, Kelynack KJ, Pedagogos E, Becker GJ. Pentoxifylline reduces in vitro renal myofibroblast proliferation and collagen secretion. Am J Nephrol 2000; 20: 82-8.

20 Chen YM, Chien CT, Hu-Tsai MI, Wu KD, Tsai CC, Wu MS, et al. Pentoxifylline attenuates experimental mesangial proliferative glomerulonephritis. Kidney Int 1999; 56: 932-43.

21 Lin SL, Chen YM, Chien CT, Chiang WC, Tsai CC, Tsai TJ. Pentoxifylline attenuated the renal disease progression in rats with remnant kidney. J Am Soc Nephrol 2002; 13: 2916-29.

22 Ramesh G, Reeves WB. TNF- $\alpha$ mediates chemokine and cytokine expression and renal injury in cisplatin nephrotoxicity. J Clin Invest 2002; 110: 835-42.

23 Zhou QG, Zheng FL, Wen YB, Tan XY, Duan L, Li Y. Altered expression of vascular endothelial growth factor and its receptors in transdifferentiated human proximal tubular epithelial cells induced by transforming growth factor betal. Zhongguo Yi Xue Ke Xue Yuan Xue Bao 2005; 27: 325-31. (Chinese).

24 Kim BS, Chen J, Weinstein T, Noiri E, Goligorsky MS. VEGF expression in hypoxia and hyperglycemia: reciprocal effect on branching angiogenesis in epithelial-endothelial co-cultures. J Am Soc Nephrol 2002; 13: 2027-36.
25 Du M, Roy KM, Zhong L, Shen Z, Meyers HE, Nichols RC. VEGF gene expression is regulated post-transcriptionally in macrophages. FEBS J 2006; 273: 732-45.

26 Bottinger EP, Bitzer M. TGF- $\beta$ signaling in renal disease. J Am Soc Nephrol 2002; 13: 2600-10.

27 Lin SL, Chen RH, Chen YM, Chiang WC, Lai CF, Wu KD, et al. Pentoxifylline attenuates tubulointerstitial fibrosis by blocking smad3/4-activated transcription and profibrogenic effects of connective tissue growth factor. J Am Soc Nephrol 2005; 16: 2702-13.

28 Chevalier RL. Molecular and cellular pathophysiology of obstructive nephropathy. Pediatr Nephrol 1999; 13: 612-9.

29 Kang DH, Kim YG, Andoh TF, Gordon KL, Suga S, Mazzali M, et al. Post-cyclosporine-mediated hypertension and nephropathy: amelioration by vascular endothelial growth factor. Am J Physiol Renal Physiol 2001; 280: F727-F736.

30 Feliers D, Duraisamy S, Barnes JL, Ghosh-Choudhury G, Kasinath BS. Translational regulation of vascular endothelial growth factor expression in renal epithelial cells by angiotensin II. Am J Physiol Renal Physiol 2005; 288: F521-529.

31 Hellwig-Bürgel T, Stiehl DP, Katschinski DM, Marxsen J, Kreft B, Jelkmann W. VEGF production by primary human renal proximal tubular cells: requirement of HIF-1, PI3-kinase and MAPKK-1 signaling. Cell Physiol Biochem 2005; 15: 99-108.

32 Nakagawa T, Lan HY, Zhu HJ, Kang DH, Schreiner GF, Johnson RJ. Differential regulation of VEGF by TGF-beta and hypoxia in rat proximal tubular cells. Am J Physiol Renal Physiol 2004; 287: F658-664. 